\title{
Continuous Visualization of P-Q Intervals in Portable Devices for Monitoring Human Organism Functional State
}

\author{
DOI: $10.17691 / \mathrm{stm} 2016.8 .1 .06$
}

Received June 11, 2015

\begin{abstract}
A.P. Kuleshov, Researcher, Department of Radio Engineering and Management Systems;
A.V. Ilyin, Researcher, Department of Radio Engineering and Management Systems;

A.P. Zaretsky, PhD, Researcher, Department of Radio Engineering and Management Systems
\end{abstract}

Moscow Institute of Physics and Technology (State University), 9 Institutskiy per., Dolgoprudny, Moscow Region, 141700, Russian Federation

An algorithm of analyzing $P-Q$ interval duration of electrocardiographic (ECG) signal enabling continuous evaluation of changes in this parameter and visualization the data in the form convenient for the analysis has been developed.

The aim of the investigation was to increase the accuracy of $P-Q$ interval recording under non-static processing conditions.

Materials and Methods. Investigations were carried out using signals obtained from the circuit board of the proprietary complex monitoring device, containing components of ECG recording. The algorithm was realized using LabView software. Not only P-Q interval but such clinically valuable parameters as scattergram area, cardiorhythmogram amplitude, recorded ECG signal spectrum, amplitude and period of respiration wave were also assessed.

Results. The suggested diagnostic criteria allow real-time analysis not only of normal ECG signal parameters with great accuracy but also pathological disorders of electrophysiological atrioventricular conduction, the complex evaluation of which is a real support for decision making in establishing a diagnosis.

Key words: algorithms of ECG signal processing; $\mathrm{P}-\mathrm{Q}$ interval assessment; Wolff-Parkinson-White syndrome; mathematical processing of ECG signals.

In recent decades there has been noted a tendency to $P-Q$ interval shortening which is increasing in number. Absolute and relative shortening of $P-Q$ interval is fixed today in more than $25 \%$ of population with maximal prevalence among young people [1,2]. Sudden death is estimated to amount to $13 \%$ among all deaths of persons younger than 30 years, it being associated in the majority of cases with cardiac causes. $\mathrm{P}-\mathrm{Q}$ interval shortening is one of the most common risk factors of sudden death at a young age [3].

Despite a high social significance and elevated danger of sudden cardiac death in the modern world, preventive measures against this pathology, complex patient examination, for example, including Holter monitoring, are conducted not to the full. It is connected with insufficient alertness of the doctors to $P-Q$ interval shortening due to a large number of cases with asymptomatic course, absence of unified examination algorithm, unawareness of the mechanism of $P-Q$ interval formation and possible prevention of atrioventricular connection (AV connection) impairment. A separate cohort with a pronounced danger of sudden death due to $P-Q$ interval shortening includes people undergoing the risk of health impairment because of their specific work.

An important role in solving this problem may belong to the improvement in hard- and software units of portable systems for recording, processing and interpretation of ECG signal by developing new methods and means of ECG processing for their application in the conditions of patients' motor activity.

The aim of the investigation is to develop an algorithm of ECG signal processing, enabling continuous analysis of $P-Q$ interval duration and its changes synchronously with visualization of the main ECG characteristics (absolute values of heart rate at a given time, relative alterations of cardiointervals, displaying respiratory activity and so on).

Materials and Methods. One of the mechanisms of a shortened $P-Q$ interval formation is an impairment of $\mathrm{AV}$ connection, manifesting itself in the reduction of pulse delay time from the sinus node [2]. Normally, $\mathrm{P}-\mathrm{Q}$ interval amounts to $0.12-0.18 \mathrm{~s}$ (up to $0.20 \mathrm{~s}$ ) and tends to become longer with age and shorter in heart rate acceleration. In average sample the greatest value of $P-Q$ interval in the examined people over 18 years is $0.20 \mathrm{~s}$, while in bradycardia it may be as long as 0.21 or even $0.22 \mathrm{~s}$. In tachycardia the $P-Q$ interval duration equals to $0.19-0.20 \mathrm{~s}$, which may be a cause of pathological changes of the electrical conduction system of the heart, e.g. early electrophysiological activation of the ventricles.

It is the dynamic assessment of the $P-Q$ interval that represents one of the most simple and convenient ways of verifying pathological changes of the electrophysiological

For contacts: Aleksey P. Zaretsky, e-mail: a.p.zaretskiy@gmail.com 
heart system (detection of additional conductive pathways or disorders of AV node conduction - AV block of various degrees). The common assessment criteria were used to analyze $P-Q$ interval:

shortening of $P-Q$ interval, the duration of which does not exceed $0.11 \mathrm{~s}$;

absence of additional excitation wave - delta-wave ( $\delta$-wave) in QRS complex;

presence of unchanged (narrow) and undeformed QRS complexes (excluding cases with the accompanied blockage of the bundle of His branches).

Change of $P-Q$ interval is one of the factors of changes in R-R interval. The main parameter of ECG analysis is heart rate on the basis of which other components of ECG signal are evaluated. A relative noncompliance of $R-R$ and $P-Q$ intervals is an important criterion of $A V$ connection functional state assessment and availability of additional conduction pathways. This noncompliance is revealed in $20 \%$ of population testifying to the initial manifestations of AV node pathology [4]. All these show the importance and clinical validity of the combined continuous visualization of $P-Q$ interval, scattergram, $R-R$ intervalogram and spirogram.

To solve the tasks of continuous visualization our original analytical module of the system for statistical electrophysiological data processing and a proprietary pilot sample of ECG recorder, comprising accelerometer, gyroscope, barometer, GPS module, humidity and temperature sensors have been used. The complex of all these units enables the device to assess electrophysiological heart activity (ECG recorder), patient's motor activity (accelerometer, gyroscope, barometer, GPS module) and the degree of physical load (humidity and temperature sensors), which is necessary for evaluation of interrelation of changes in $P-Q$ and $\mathrm{R}-\mathrm{R}$ interval duration.

The following functional capabilities distinguish the developed device from similar systems:

1. Recording of the ECG signal parameters in the examined person, as well as on-line evaluation of $P-Q$ interval and the analysis of their correlation with the readings of accelerometer, gyroscope and electrodermography sensors allow the assessment of compensatory body function (detection of heart rate change states not associated with physical activity) and interrelated changes of $P-Q$ and $R-R$ interval duration.

2. Continuous monitoring of patient location for timely arrival of the emergency team.

3. Improved technology of noise-proof registration of sensor readings (data on the cardio-respiratory system state, motor activity, electrodermal reaction, patient's body temperature and blood pressure) based on the improved algorithms of analog and digital filtration of signals.

4. Optimized overall dimensions of the device for wireless transmission of the recorded parameters to the system supporting decision making.

5. Extensive processing of the recorded signal by the server for detailed evaluation of cardio-respiratory system condition.

Building of the model of the heart rate variability microstructure and $P-Q$ interval included automatic recording of the successive cardiointervals in the intensity index standard lead under moderate motor activity conditions (Figure 1). The duration of the demonstrated signal area, selected from the general continuous signal, was $20 \mathrm{~s}$. The criterion of duration selection was the convenience of visualization, however the interface of the developed program allows the isolation of the random time length signals.

The initial stage of real-time working with ECG is the analysis of the recorded electrical cardiac activity data. It should be noted that discretization frequency of the signal must not be below $125 \mathrm{~Hz}$, because frequencies up to $25 \mathrm{~Hz}$ are present in the useful range of ECG signal frequencies obtained in the in- and outpatient environment $[3,4]$. Under the hypothesis of Kotelnikov's theorem [5] the discretization frequency, used in the study, was set 5 times greater than the maximum signal frequency. The results of the analysis have demonstrated, that increase of discretization frequency in comparison with the selected one leads to the unreasonable increase of signal samples, resulting in the software overload and also noise enhancement.

Primary signal processing includes signal visualization in the program medium, which enables real-time

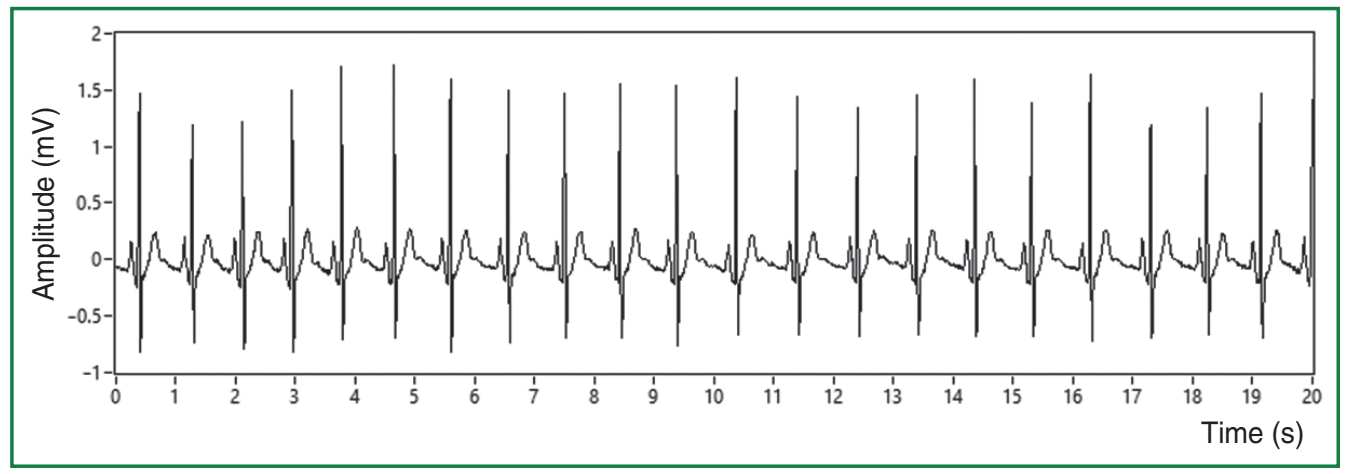

Figure 1. Electrocardiogram selected for the analysis 
monitoring of the pathological states. Continuous visualization of the spectral constituent, obtained after Fourier transformation, is certain to be important when working with any bioelectrical signal (Figure 2). The spectrum derived allows the assessment of the signal frequency range and making a decision on setting digital filtering parameters of the received signal. The study results has clearly demonstrated that useful signal frequencies include the range up to $25 \mathrm{~Hz}$, while the rest constituent may be classified as a high-frequency interference and its filtration may be carried out.

At the next stage digital signal filtering is performed. For this purpose a Butterworth passband filter was chosen as one of the most widely used, stable in operation and having the "flattest" possible amplitudefrequency characteristic compared to its analogs. To realize filtration the program is able to set the upper and lower cut-off frequency. As mentioned above, frequencies above $25 \mathrm{~Hz}$ (this value is chosen as a cutoff frequency to eliminate high-frequency interferences) are to be removed from the signal. A cut-off frequency for low-frequency interferences was chosen taking into consideration the effect of the respiratory activity on the ECG signal recording (Figure 3) [6, 7].

An important stage of ECG signal evaluation is counting $R$ waves in the mode of continuous visualization enabling the assessment of regularity and rate, which are important criteria of pathology presence or absence. It is important to note, that the signal after filtration does not contain other peaks except $P, R$ and $T$. The algorithm, created within the frames of the study, in contrast to the existing analogs, makes it possible to isolate the mentioned peaks with an accuracy of more than $93 \%$, making the task of $P-Q$ interval isolation more successful [8].

A search algorithm consists in the comparison of $i$-th signal value, $i-1$ and $i+1$ values. If $i$ value is more than the adjacent values, it is considered a peak and is recorded to the dynamic value array, which has a periodic structure. $\mathrm{R}$ wave is characterized by the maximal amplitude, therefore the application of the given algorithm for peak search in the formed value array is grounded. Working with the algorithm, it was found, that $R$ waves were detected with $100 \%$ accuracy. If there are

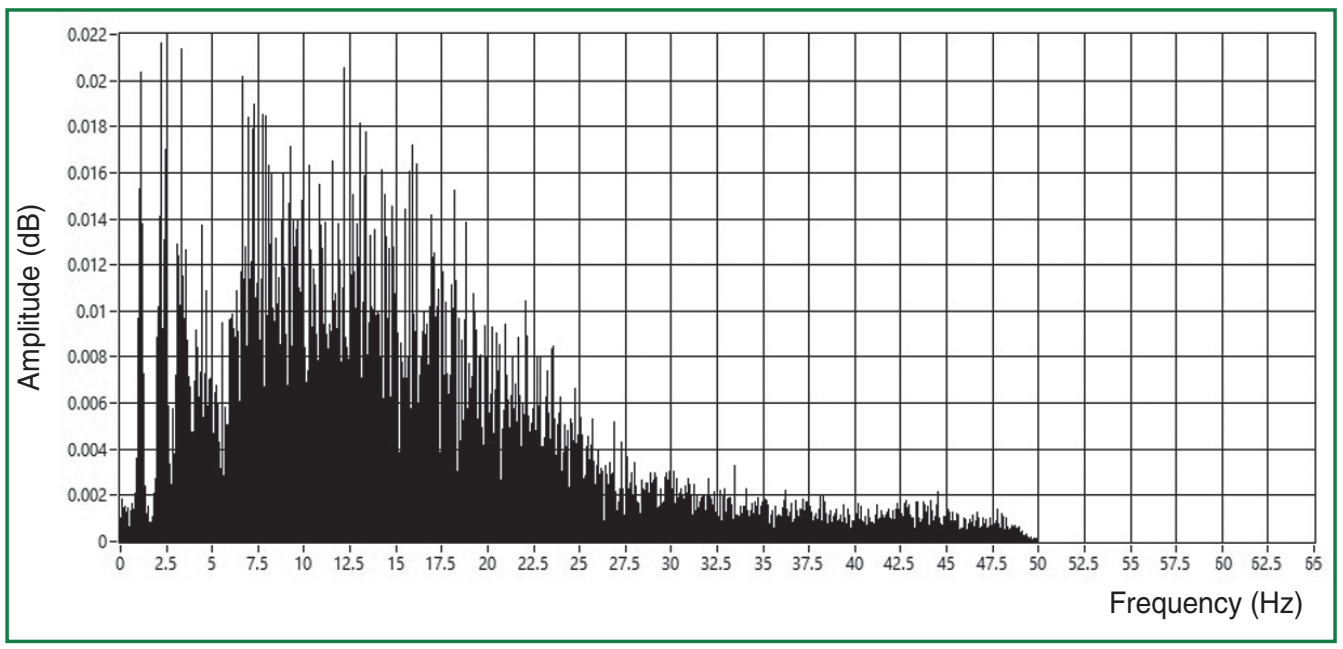

Figure 2. Spectrum of the analyzed ECG signal

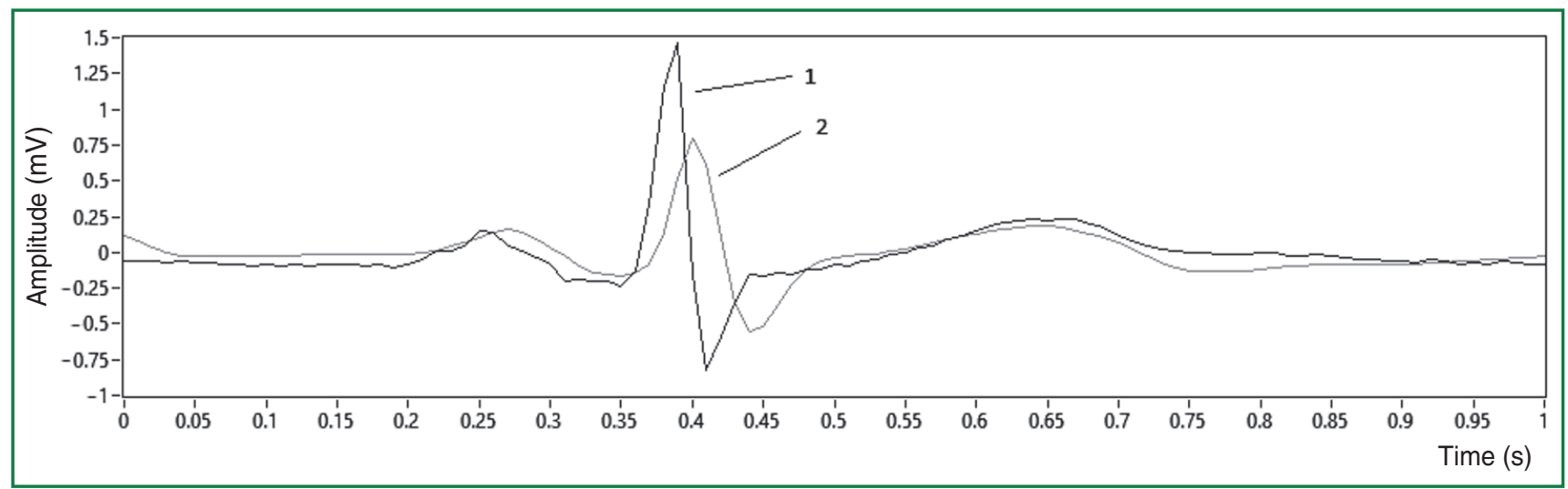

Figure 3. The analyzed ECG signal before (1) and after (2) filtration 
adjacent $R$ waves located successively, the first array of the obtained peaks is compared with the maximal value in the signal. The difference of maximal amplitude value and the current $R$ wave amplitude will be less than the wave amplitude, which helps to avoid detection error. Thus, there is no need to choose threshold values to determine the peak, which is the key advantage of the presented algorithm of $\mathrm{R}$ wave detection.

The main information on the condition of the systems, regulating the heart rate, is contained in the "characteristics of dispersion" of cardiointerval durations (Figure 4). When analyzing the heart rate variability it is necessary to assess the presence of sinus tachycardia, reflecting complicated processes of interaction of various heart rate regulation contours, by comparing $\mathrm{R}-\mathrm{R}$ interval duration with the values of the conventional "norm" for the given sex-age group. If rhythm disturbances of diverse origin are present, application of special methods for restoration of the studied process stationarity are required or it is necessary to use special analytical approaches.

$R-R$ interval is calculated by subtraction of the time value of the current $R$ interval from the value of the previous one. It is convenient to analyze the cardiointervalogram by means of the histogram of $R-R$ interval distribution, i.e. a bar chart in which the height of each column corresponds to the quantity of R-R intervals fallen into time range of the column considered. Once the bar chart is built, its range, mode and mode amplitude, used to evaluate rhythm stability, are found using criterion of intensity index (II), which is measured in conventional units and is expressed by a ratio

$$
\mathrm{II}=\frac{A M o}{3 \cdot M o \cdot D_{x}},
$$

where Mo (mode) - the most frequently occurring value of $\mathrm{R}-\mathrm{R}$ interval duration in seconds; $A M o$ (mode amplitude) - a number of interval values equal to Mo in percent to the total number of the recorded cardio- cycles; $D_{x}$ (variation range) - the difference between the maximal and minimal duration value of the recorded $\mathrm{R}-\mathrm{R}$ intervals in seconds.

The formula presented for intensity index calculation allows the extension of this assessment method to the age group over 18 years in contrast to other traditional analogs. The diagnostic significance of intensity index criterion may be presented in the following way: intensity index up to 70 units - stability of rhythm; intensity index over 70 units - instability of rhythm caused by overstrain of the regulatory systems.

Detection of $R-R$ intervals makes it possible to build a scattergram (Figure 5), which is a much more compact way of rhythm visualization relative to cardiorythmogram. This method is especially useful for those cases when rare and sudden events occur amid the monotonous rhythm.

Signals in the LabVeiw program medium are obtained by subtracting the array of $R$ wave time values from the same array shifted one element back, which enables, in contrast to the existing analogs, the calculation of the correlation coefficients when a signal is shifted by one cycle similar to the building of correlation rhythmogram [9]. Thus, when building a correlation rhythmogram the points of it generate a population with the center lying on the bisector. The distance from the center to the beginning of the reference axis corresponds to the most expected duration of the cardiac cycle.

No doubt, evaluating the electrical cardiac activity, it is necessary to touch upon the questions of the respiratory regulation of the mechanical heart activity, the solution of which lies in the analysis of the respiratory wave. To select the respiratory component from the general signal, it is necessary to average in each sample the filtered signal in the frequency range of $0-0.5 \mathrm{~Hz}$, obtained with the help of low-frequency Butterworth filter. Simultaneously with such conversions respiratory rate, the changes in which are constantly visualized, is also calculated, making it possible

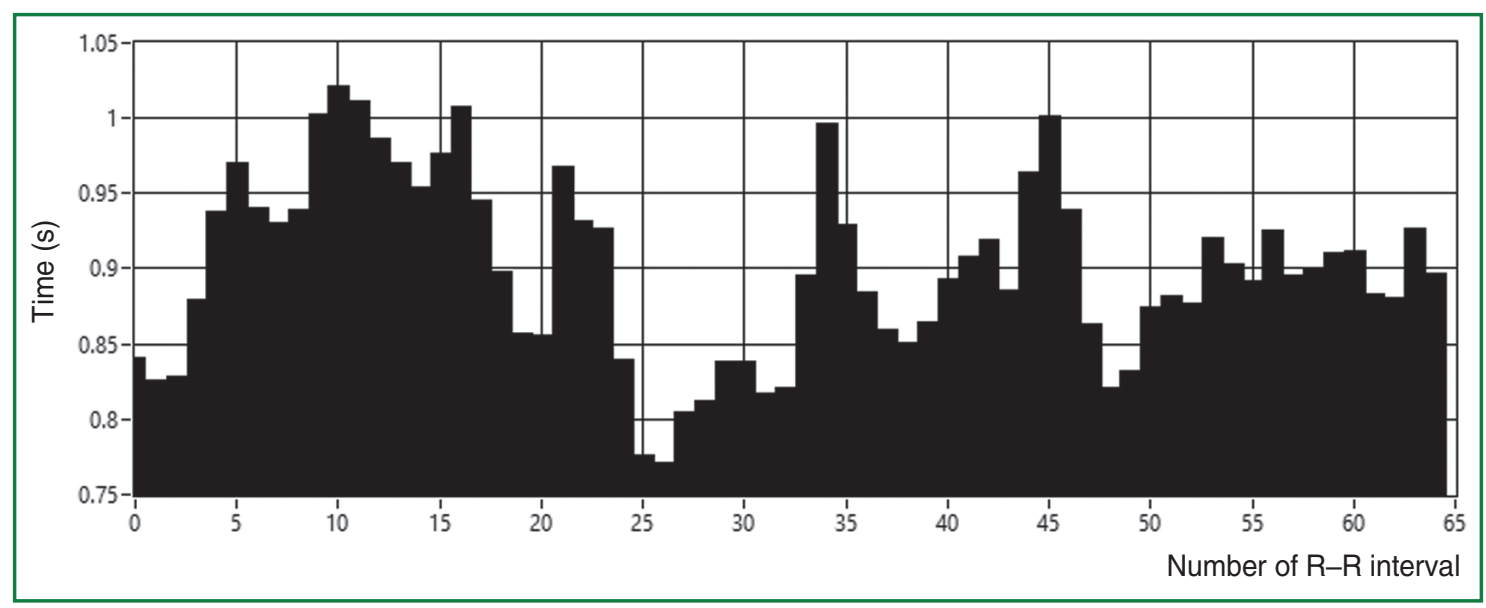

Figure 4. Cardiointervalogram of the analyzed ECG signal 


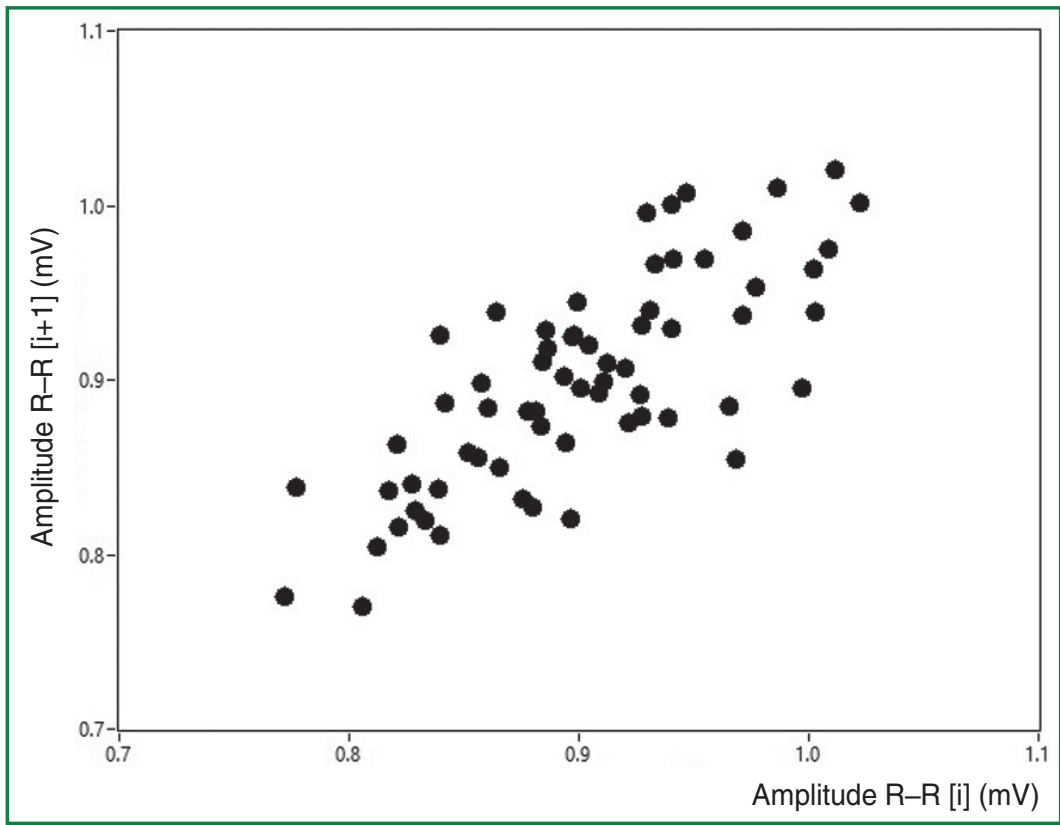

Figure 5. Scattergram of the analyzed ECG signal

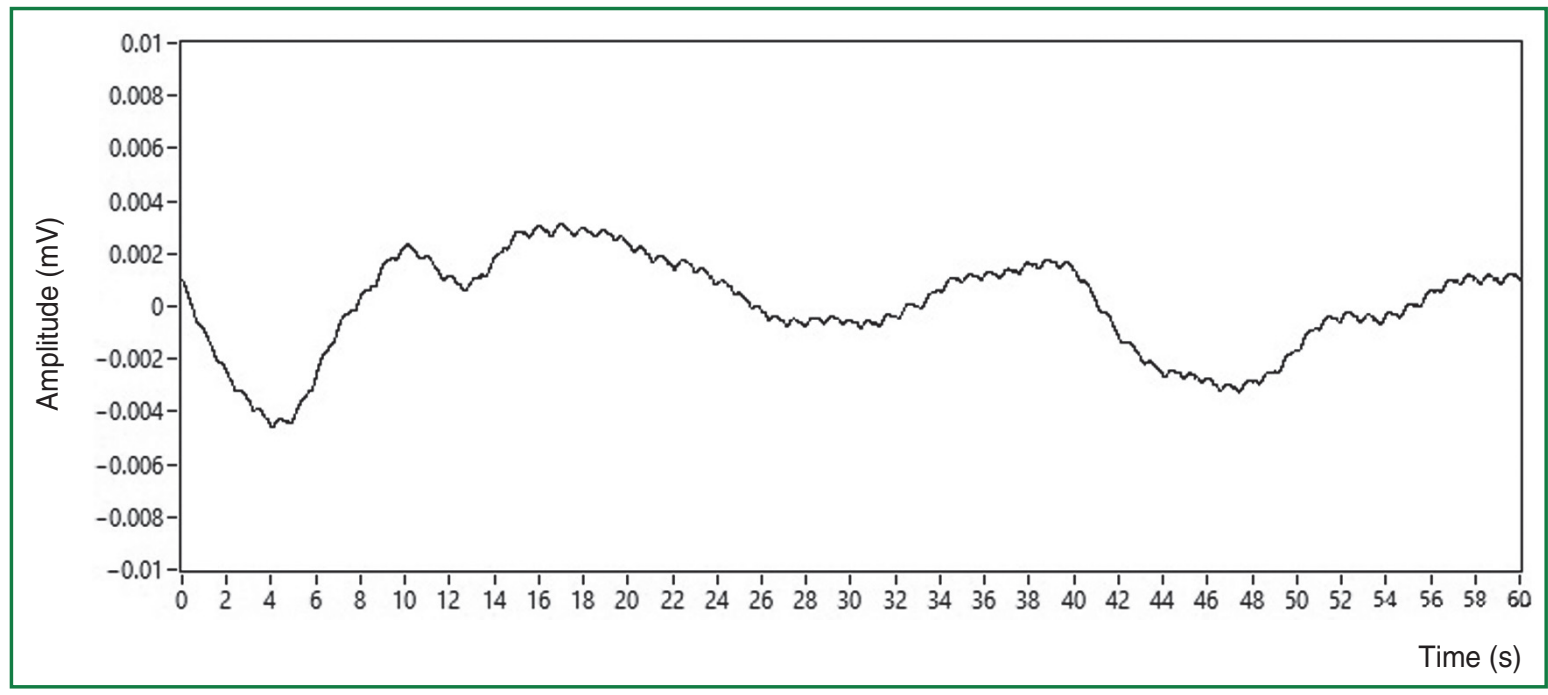

Figure 6. Spirogram selected from the analyzed ECG signal

to combine electrophysiological and respiratory constituent assessment (Figure 6).

During the next stage the main task of the present investigation is being solved - separation and assessment of $P-Q$ interval. Duration of $P-Q$ interval is determined according to the following algorithm:

1) calculation of the first signal derivative at which peak values of the signal become zero, and zero values become peaks; the highest peak is observed in $Q$ wave;

2) recording the time $t_{Q}$ of $Q$ wave and calculation of its amplitude $A_{Q}$ by ECG signal;

3) automatic setting of a threshold value the amplitude $A$ to find the beginning and end of $Q$ wave as the difference of $\mathrm{Q}$ and $\mathrm{P}$ amplitudes: $A=\left(A_{Q}-A_{P}\right) / 2$;

4) computing the time of $P$ wave end;

5) subtraction of $Q$ wave time $t_{Q}$ from the selected time of the P-wave end $t_{P}: P-Q=t_{Q}-t_{P}$.

A point with duration of $P-Q$ interval is built for each number of $\mathrm{R}-\mathrm{R}$ interval, which actually corresponds to the number of $\mathrm{P}-\mathrm{Q}$ interval (Figure 7).

The algorithm presented makes it possible, in contrast to the analogs, to increase the accuracy of determining P-Q interval duration by $11 \%$ owing to the preliminary digital filtration of the signal, which, in its turn, improves the reliability of determining the time of $P$ wave end and beginning of $Q$ wave. 


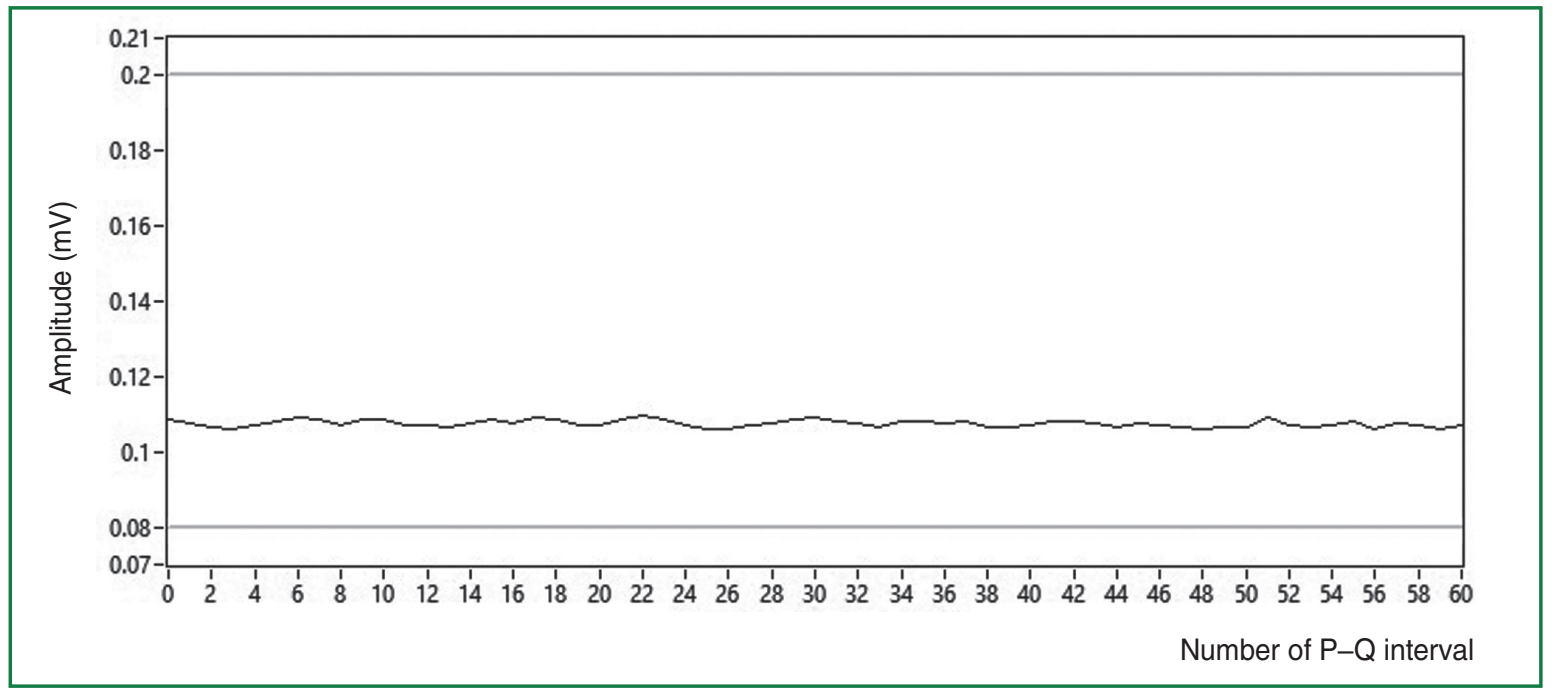

Figure 7. Monitoring of $\mathrm{P}-\mathrm{Q}$ intervals of the analyzed ECG signal (grey color denotes the borders of the norm)

Discussion. Continuous visualization of $\mathrm{P}-\mathrm{Q}$ interval duration allows the user to clearly evaluate the deviation of the current interval duration from the conventional norm. When interpreting the results, the following classification of the pathological atrioventricular conduction is used:

1. First-degree AV block:

all atrium impulses reach the ventricles (conduction rate 1:1); but the conduction through the AV node occurs with an equal delay;

values of $P-Q$ intervals go beyond the "upper" border of the norm on the diagram not more than 3 times during recording of $60 \mathrm{QRS}$ complexes.

2. Second-degree AV block:

single atrium impulses are not conducted to the ventricles (conduction 2:1);

values of $P-Q$ interval duration go beyond the "upper" border of the norm on the diagram not more than 12 times during recording of $60 \mathrm{QRS}$ complexes.

\section{Third-degree AV block (full AV block):}

impulses from the atria do not reach the ventricles, complete mismatch of the atria and ventricular rhythms;

values of $P-Q$ intervals are constantly over the "upper" border of the norm of $0.2 \mathrm{mV}$ during a long-time recording.

\section{Wolff-Parkinson-White syndrome:}

values of $P-Q$ intervals are constantly below the "lower" border of the norm equal to $0.08 \mathrm{mV}$ during a long-time recording. In this case intensity index is less than 70 units.

Thus, the software developed for the analysis of $\mathrm{P}-\mathrm{Q}$ interval changes, based on the presented algorithms, allows the user to verify successfully the diseases of the cardiac conduction system.

Conclusion. The developed algorithm of R-wave detection, which, in contrast to its analogs, does not use subjective threshold values, as well as the algorithm of determining $P-Q$ interval duration, differing from the analogs by a convenient method of detecting the time of $P$ and $Q$ wave ends, will increase the accuracy of arrhythmia diagnosis by $11 \%$ in comparison with the similar methods used. Separation and the following analysis of the main components of ECG signal with the help of the created algorithms and means of continuous visualization of values of $P-Q$ intervals in portable devices of monitoring functional state of the human body make the number of false results minimal.

Study Funding. The development was supported by the subsidy of the Ministry of Education and Science of the Russian Federation (unique project identifier RFMEFI57814X0052).

Conflicts of Interest. The authors declare no conflicts of interest.

\section{References}

1. Berezhnyi V.V., Marushko T.V. Sudden death during physical exercises at children and adolescents. Sovremennaya pediatriya 2009; 6(28): 29-34.

2. Vorobejv L.V. Index PQs, as evidence risk of heart rhythm disturbance tachycardia. Uspekhi sovremennogo estestvoznaniya 2013; 11: 8-13.

3. Vorobejv L.V. Shortening of the P-Q, accents ECG diagnostics. Sovremennye naukoemkie tekhnologii 2013; 11: 152-157.

4. Zimetbaum P.J., Mark E.J. Practical clinical electrophysiology. Lippincott Williams and Wilkins; 2008.

5. Kotel'nikov V.A. On air and wire transmission capacity in telecommunications. All-Union Energy Committee. Materials for the First All-Union Congress on reconstruction of communication facilities and development of low-current industry, 1993. Uspekhi fizicheskikh nauk (reprint) 2006; 176(7): 762-770.

6. Bogomolov A.V., Maistrov A.I. Theoretical-experimental 
analysis of convergence of heart rate variability spectral measures estimated via heart rate and heart period signals. Biomedical Engineering 2009; 43(2): 75-80, http://dx.doi. org/10.1007/s10527-009-9091-y.

7. Zaretskiy A.P., Kuleshov A.P., Alekhin M.D. Analiz variabel'nosti serdechnogo ritma patsientov $s$ zheludochkovymi narusheniyami pri vremennoy elektrokardiostimulyatsii. $\mathrm{V} \mathrm{kn}$.: Materialy Vserossiyskoy nauchno-prakticheskoy konferentsii $s$ mezhdunarodnym uchastiem "Variabel'nost" serdechnogo ritma: teoreticheskie i prikladnye aspekty" [Analysis of heart rate variability in patients with ventricular disorders in temporal electrocardiostimulation. In: Materials of the All-Russian Scientific and Practical Conference with international participants "Heart rate variability: theoretical and applied aspects"]. Cheboksary; 2014; p. 66-69.

8. Kukushkin Y.U., Maistrov A.I., Bogomolov A.V. Rhythmocardiogram approximation methods for calculation of spectral parameters of cardiac rhythm variability. Biomedical Engineering 2010; 44(3): 15-30, http://dx.doi.org/10.1007/ s10527-010-9165-x.

9. Zaretskiy A.P., Ilyin A.V., Kuleshov A.P., Poteryakhina A.V., Poteryakhin A.V. Features of analysis and daily registration of ECG in patients with paroxysmal atrial fibrillation. Biol Med (Aligarh) 2015; 7(2): BM-098-15. 